$5-6$

\title{
Medical informatics: the future of organized healthcare or Orwellian medicine?
}

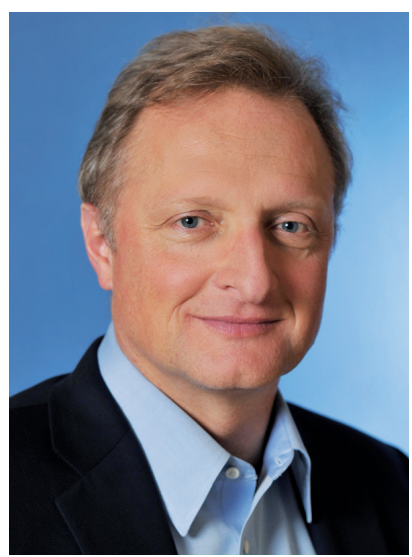

Jens Chapman
Public healthcare information technologies or 'medical informatics' may very well become the preeminent medical development of the 21 st century, akin to the breakthrough role antimicrobial therapies played starting in the late 19th century and the impact that genomics were starting to have in the 20th century. If current trends prevail, large-scale data management systems, fueled by the combination of rapid developments in medical information systems and rapidly decreasing costs for data storage and retrieval, will be connected almost directly to all but the most remote or destitute of medical practices anywhere in the world.

Currently, United States government agencies collect a wide variety of hospital discharge data on most inpatients. With such administrative datasets, it is then up to the holder of the data to determine how our practices are rated and what support we will receive for our care delivery in the future. Never mind that some items such as 'hyponatremia' (one of the currently collected screening values) will likely have little or next to nothing to do with the quality of care that a spine surgery patient may have received. If a patient turns up 'hyponatremic' on a federal discharge database it results in a demerit for the practitioner and the hospital without chance for appeal or explanation.

Are current practices, such as recording a sodium level, the harbinger of quality of care for remote specialties such as spine surgery in the future? Probably not, but it isn't all that farfetched to imagine comprehensive data sets being gathered to screen our practice patterns. The sophistication of data gathering will undoubtedly grow, allowing for identification of blemishes and real complications alike in an unprecedented fashion. From there it is but a small step to envision future healthcare systems that are driven by data analysis of apparent 'evidence' to decree treatment decisions based on abstract principles without the power of human touch. This is the tipping point where the currently popular, but largely irrelevant 'guidelines' (usually turned out by professional societies) will be rendered completely irrelevant by implementation of healthcare 'policies' decreed by governmental organizations and powerful third-party payor corporations. This vision is very much in keeping with the dystopian prophecies of author George Orwell, perhaps most well known for his bestsellers "Animal Farm" written in 1945 and "Nineteen EightyFour" written in 1949, who predicted healthcare being administered through a remote healthcare bureaucracy that took it upon itself to decide what was best for its citizens.

The current, undeniably fascinating, leap in information technology could indeed result in the realization of the Orwellian nightmare: healthcare decreed by 'careless' anonymous bureaucratic calculation with, human disease and its treatment reduced to a formula and issued in the form of a mandated decision.

Aside from this nightmarish scenario-are there some potential benefits for patient care inherent to a progressive implementation of healthcare informatics? Sure! There are five major points that immediately come to mind:

- Rare diseases will become much more transparent with modes of detection and treatment as long as they are well-identified a priori in large-scale data gathering. 
- Comparison of certain complication rates and treatment outcomes of larger populations will become more feasible.

- General trends and shifts in healthcare utilization can be detected for common ailment categories treated in consistent fashion.

- Accounting tools and public health planning will be improved to the benefit of lowering costs of healthcare to society.

- Social engineering and public information campaigns may be implemented to prevent disease in the first place.

These insights gained by health informatics are undeniably valuable to society, but how can we prevent abuse by having general insights collectively applied in the decisionmaking process for individual patients?

At the very heart of this ethically charged debate lies the concept of properly practiced evidence based medicine (EBM). From its very inception, EBM, has been defined as the "conscientious, explicit, and judicious use of the current best evidence in making decisions about the care of individual patients." [1] It is this emphasis on the 'individual' that makes EBM so powerful and gives it its ethical foundation. EBM in the eyes of its original describers was never intended to be "cookbook" medicine prescribed for the masses at the cost of ignoring the individual—be it a patient or a care provider.

Of course medical informatics will be a force to be reckoned with. But it is up to us, the providers on the ground, to make the connections of medical informatics to the truly affected individuals-our patients. It is up to us to identify what makes our patients unique and then define these unique characteristics so that they become a visible part of the data gathered and outlier patients can be better identified. The article by Arnold et al (pages 11-15) in this issue is one of the many necessary steps in the right direction. Although of lower-evidence base (it is a retrospective case-series), this honest, comprehensive gathering of consecutive data from patients undergoing routine anterior neck surgery by one surgeon using the same care paths for all his patients has shown surprisingly wide discrepancies in one commonly collected metric: time to discharge. It is now up to us, the medical experts on the ground to look at the causes for these variances in greater detail.

AOSpine offers you a number of unique resources toward finding answers in this age of medical informatics:

1. Knowledge Forum: Advancing the knowledge of spine care with an integrative approach of combining research and education.

2. EBSJ: Enhancing the quality of research and its reporting.

3. AO CID: Proactively creating systems for meaningful data collection, analysis, reporting, and synthesis.

1. Sackett DL, Rosenburg WMC, Gray JAM (1996) Evidence-based medicine: what it is and what it isn't. BMJ; 312:71-76.
By themselves and collectively AOSpine resources can provide you with the tools to detect and better describe the individuality of our patients and provide us all with better evidence to support our care decisions in the future.

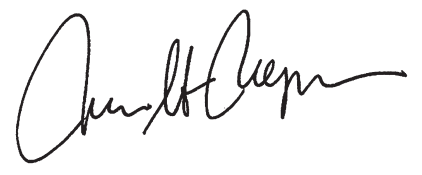

Jens Chapman, Editor-in-Chief 